\title{
The nasal delivery of nanoencapsulated statins - an approach for brain delivery
}

\author{
This article was published in the following Dove Press journal: \\ International Journal of Nanomedicine \\ 7 December 2016 \\ Number of times this article has been viewed
}

\author{
Adryana Clementino ${ }^{1,2}$ \\ Mellissa Batger ${ }^{3}$ \\ Gabriela Garrastazu ${ }^{2,3}$ \\ Michele Pozzoli ${ }^{3}$ \\ Elena Del Favero ${ }^{4}$ \\ Valeria Rondelli ${ }^{4}$ \\ Bianca Gutfilen ${ }^{5}$ \\ Thiago Barboza ${ }^{5}$ \\ Maria B Sukkar ${ }^{3}$ \\ Sergio A L Souza ${ }^{5}$ \\ Laura Cantù ${ }^{4}$ \\ Fabio Sonvico ${ }^{1,3}$ \\ 'Department of Pharmacy, \\ University of Parma, Parma, Italy; \\ ${ }^{2}$ National Council for Scientific and \\ Technological Development - CNPq, \\ Brasilia, Brazil; ${ }^{3}$ Graduate School of \\ Health - Pharmacy, University of \\ Technology Sydney, Ultimo, NSW, \\ Australia; ${ }^{4}$ Department of Medical \\ Biotechnologies and Translational \\ Medicine, LITA, University of Milan, \\ Segrate, Italy; ${ }^{5}$ Laboratório de \\ Marcação de Células e Moléculas, \\ Department of Radiology, Faculty of \\ Medicine, Federal University of Rio \\ de Janeiro, Rio de Janeiro, Brazil
}

Correspondence: Fabio Sonvico

Graduate School of Health - Pharmacy,

University of Technology Sydney,

Building 7, Level 4, 67 Thomas Street,

PO Box 123, Ultimo, NSW 2007,

Australia

Tel +6I 295 I4 8303

Email fabio.sonvico@uts.edu.au
Purpose: Along with their cholesterol-lowering effect, statins have shown a wide range of pleiotropic effects potentially beneficial to neurodegenerative diseases. However, such effects are extremely elusive via the conventional oral administration. The purpose of the present study was to prepare and characterize the physicochemical properties and the in vivo biodistribution of simvastatin-loaded lecithin/chitosan nanoparticles (SVT-LCNs) suitable for nasal administration in view of an improved delivery of the statins to the brain.

Materials and methods: Chitosan, lecithin, and different oil excipients were used to prepare nanocapsules loaded with simvastatin. Particle size distribution, surface charge, structure, simvastatin loading and release, and interaction with mucus of nanoparticles were determined. The nanoparticle nasal toxicity was evaluated in vitro using RPMI 2651 nasal cell lines. Finally, in vivo biodistribution was assessed by gamma scintigraphy via Tc99m labeling of the particles.

Results: Among the different types of nanoparticles produced, the SVT-LCN_MaiLab showed the most ideal physicochemical characteristics, with small diameter $(200 \mathrm{~nm})$, positive surface charge $(+48 \mathrm{mV})$ and high encapsulation efficiency (EE; 98\%). Size distribution was further confirmed by nanoparticle tracking analysis and electron microscopy. The particles showed a relatively fast release of simvastatin in vitro (35.6\% $4.2 \%$ in 6 hours) in simulated nasal fluid. Blank nanoparticles did not show cytotoxicity, evidencing that the formulation is safe for nasal administration, while cytotoxicity of simvastatin-loaded nanoparticles $\left(\mathrm{IC}_{50}\right)$ was found to be three times lower than the drug solution $(9.92$ vs $3.50 \mu \mathrm{M})$. In rats, a significantly higher radioactivity was evidenced in the brain after nasal delivery of simvastatin-loaded nanoparticles in comparison to the administration of a similar dose of simvastatin suspension.

Conclusion: The SVT-LCNs developed presented some of the most desirable characteristics for mucosal delivery, that is, small particle size, positive surface charge, long-term stability, high EE, and mucoadhesion. In addition, they displayed two exciting features: First was their biodegradability by enzymes present in the mucus layer, such as lysozyme. This indicates a new Trojan-horse strategy which may enhance drug release in the proximity of the nasal mucosa. Second was their ability to enhance the nose-to-brain transport as evidenced by preliminary gamma scintigraphy studies.

Keywords: nose-to-brain, simvastatin, nanoparticles, neurodegenerative diseases, gamma scintigraphy, small-angle X-ray scattering (SAXS), lysozyme, biodegradable nanoparticles

\section{Introduction}

The 3-hydroxy-3-methylglutaryl coenzyme A (HMG-CoA) reductase inhibitors, or statins, are arguably among the biggest advances in cardiovascular care in the 20th century. Statins reduce cholesterol serum levels by reversibly inhibiting HMGCoA reductase, an essential enzyme in cholesterol biosynthesis, reducing the risk of serious cardiovascular events. ${ }^{1,2}$ Along with their lipid-lowering effects, statins have 
been credited for a range of outcomes or "pleiotropic effects". 3 The mechanisms by which pleiotropic outcomes occur are diverse and still not fully elucidated. Many of those effects are attributed to the inhibition of isoprenoid intermediates, that is, farnesyl pyrophosphate and geranylgeranyl pyrophosphate, and their downstream effects on intracellular signaling proteins Ras, Rho, and Rac. ${ }^{4}$ Pleiotropic effects of statins include anti-inflammatory, antioxidant, immunomodulatory, and antithrombotic actions as well as the ability to stabilize atherosclerotic plaques and inhibit the proliferation of vascular smooth muscle. ${ }^{5-7}$ Because of these pleiotropic effects, it is now believed that statins could be more widely employed in other diseases, such as rheumatoid arthritis, COPD, cancer, and neurodegenerative disorders. ${ }^{8-11}$

In the case of Alzheimer's disease, clinical research evidenced that an increase in brain cholesterol levels directly upregulates the production of $\beta$-amyloid protein, the major protein involved in the formation of senile plaques in the brain of Alzheimer's patients. ${ }^{12,13}$ Moreover, the most widely recognized risk factor of late-onset Alzheimer's is the genetic variation in a transporter of cholesterol called apolipoprotein E $\varepsilon 4$ which supposedly alters the brain cholesterol homeostasis, leading to Alzheimer's disease development. ${ }^{14,15}$ The inhibition of brain cholesterol synthesis has been shown to reduce $\beta$-amyloid accumulation, interfering with the production of $\beta$-amyloid and its accumulation as extracellular plaques. ${ }^{16,17}$ These works suggest that the effects of statins in lowering the cholesterol levels may have a beneficial role on the pathogenesis of Alzheimer's disease. It has also been postulated that statin pleiotropic effects could provide further benefit to Alzheimer's patients via modulation of the chronic inflammatory response, another key factor in neurodegenerative process. ${ }^{11}$ However, these effects of statins are only seen at high therapeutic concentrations at the target organ, and they are difficult to be observed when the conventional oral administration route is selected. In fact, statins undergo extensive first-pass metabolism, and their hydrophilic metabolites are prevented from crossing the blood-brain barrier (BBB), the principal biological barrier protecting the central nervous system (CNS). ${ }^{3}$ Despite the fact that statins are generally well tolerated, this drug class has been associated with some adverse events, in particular myopathy. This side effect can be severe and progress to rhabdomyolysis, to the point that cerivastatin was withdrawn from the market in 2001 as a consequence of 52 drug-related fatalities worldwide. ${ }^{18}$

In the last few decades, the nasal mucosa has been demonstrated to be a site for drug administration that could allow for fast and efficient absorption of drug molecules normally not suitable for oral administration. ${ }^{19}$ More recently, the intranasal route has been increasingly investigated to deliver drugs to the brain aimed at the treatment of specific brain diseases, including neurological diseases, such as Parkinson's, schizophrenia, epilepsy, and Alzheimer's. ${ }^{20}$ Several research suggest that the "nose-to-brain" route is one of the most important developments of pharmaceutical research in brain treatment, including the following: i) the potential to avoid gastrointestinal (GI) and hepatic first-pass metabolism; ii) the possibility of delivering drugs not suitable for oral administration, such as peptides and proteins; and iii) most importantly, the transport of exogenous material directly from the nasal cavity to the brain, thus bypassing the $\mathrm{BBB} \cdot{ }^{21,22}$ It is known that the unique physiology of the olfactory region within the nasal cavity can provide a direct route of administration to the $\mathrm{CNS}$, through the main innervation of the nasal cavity, that is, the olfactory and trigeminal nerves. ${ }^{23,24}$ However, drug delivery via the nose is also limited by a number of factors such as the administration volume, the barrier of the nasal epithelium, the nasal metabolic activity and the presence of a protective mucus layer. Additionally, the amount of drug administered nasally which has been shown to be transported directly from nose-to-brain is very low, normally $<0.1 \%$. Hence, the system is not currently used in clinical practice. ${ }^{25}$

The extent of the nose-to-brain drug absorption has been shown to be highly dependent on the drug formulation. ${ }^{26,27}$ The strategy of administering drugs encapsulated in nanoparticles via the olfactory epithelium could potentially improve the direct CNS delivery. Nanoparticles can improve nose-tobrain drug delivery, since they are able to interact with the nasal epithelium enhancing the drug absorption, protect the encapsulated drug from biological/chemical degradation and avoid the drug transport to the extracellular space by efflux proteins, such as P-glycoprotein. This could potentially increase CNS availability of the drug. In addition, their small diameter potentially allows for transcellular transport of nanoparticles through olfactory neurones to the brain, via the various endocytic pathways of neuronal cells in the olfactory region. ${ }^{21}$

From a drug delivery perspective, polymeric nanoparticles have proven to perform statistically better in delivering model drugs into CNS, in enhancing their pharmacological activity and/or reducing side effects, when compared to traditional formulations of drugs, when administered intranasally. ${ }^{28,29}$ Colloid nanoparticles, composed of polysaccharides, such as chitosan, and phospholipids, have been proposed recently 
as a biocompatible, biodegradable, and safe delivery system for poorly soluble drugs in order to overcome biological barriers. ${ }^{30,31}$ Their chitosan surface layer in particular has the potential to facilitate nasal delivery, due to the polysaccharide mucoadhesive properties and its potential to increase epithelial permeability by interaction with the junctional complexes between cells. ${ }^{32,33}$ In addition, this approach could potentially reduce classical statin side effects as nasal delivery allows dosage reduction and systemic exposure to the drug.

In the present study, a mucoadhesive formulation based on self-assembled lecithin/chitosan nanoparticles (LCNs) designed for intranasal administration was developed and optimized as a promising strategy for delivering simvastatin into the CNS. Desired features of this approach were high positive charge, small particle size and high drug content. In order to obtain these features, different oils were incorporated into LCNs in order to optimize the formulation. Physical and chemical stabilities were assessed along with drug release in simulated nasal fluid. Nanoparticle structure and interaction with mucus with and without lysozyme were investigated as well. The in vitro nasal toxicity of the nanoparticles alone or loaded with simvastatin was evaluated in a human nasal cell line (RPMI 2651). Finally, a preliminary gamma scintigraphy study of the biodistribution of simvastatin-loaded nanoparticles in vivo after intranasal instillation was carried out in rats.

\section{Materials and methods Materials}

Chitosan (Chitoclear FG, deacetylation degree 95\%, viscosity $45 \mathrm{cP}$ ) was provided by Primex (Siglufjordur, Iceland) and used without further purification. Lecithin (Lipoid S45) was obtained from Lipoid AG (Ludwigshafen, Germany). Pharmaceutical-grade oils Maisine ${ }^{\mathrm{TM}}$ 35-1 (glycerol monolinoleate), Labrafac ${ }^{\mathrm{TM}}$ Lipophile WL 1349 (medium-chain triglycerides, European Pharmacopoeia), Capryol ${ }^{\mathrm{TM}}$ PGMC (propylene glycol monocaprylate type I, National Formulary $[\mathrm{NF}]$ ), and Capryol ${ }^{\mathrm{TM}} 90$ (propylene glycol monocaprylate type II, NF), were a kind gift of Gattefossé (Saint-Priest, France). Simvastatin USP 99\%, mucin from porcine stomach (type III), human lysozyme and bovine serum albumin (BSA) were supplied by Sigma-Aldrich (St Louis, MO, USA).

Centrifugal filter devices (Vivaspin ${ }^{\circledR}$ 2; 30,000 molecular weight cut-off [MWCO] HY) were obtained from Sartorius (Göttingen, Germany). Dialysis tubing cellulose (14,000 MWCO) was supplied by Sigma-Aldrich. Cell line RPMI 2650 (CCL-30) was purchased from American Type Culture Collection (ATCC) (Manassas, VA, USA). Minimum essential medium (MEM) and fetal bovine serum (FBS) were acquired from Thermo Fisher Scientific (Waltham, MA, USA). Cell culture inserts and other culture plastics were from Corning Incorporated (Corning, NY, USA). All other chemicals were of analytical grade. Ultrapure and degassed ultrapure water (Purelab Flex; ELGA-Veolia LabWater, Windsor Court, UK) was used in all experiments.

\section{Methods}

\section{Preparation of simvastatin-loaded LCNs}

Simvastatin-loaded (SVT) LCNs were prepared as reported previously with slight modifications. In summary, $4 \mathrm{~mL}$ of an ethanol solution containing soybean lecithin $(100 \mathrm{mg})$, simvastatin $(50 \mathrm{mg})$ and different types of oils (Maisine [Mai], Labrafac [Lab], Capryol PGMC [Cap $]$ ], and Capryol $\left.90\left[\mathrm{Cap}_{\mathrm{II}}\right]\right)$ in $1: 1$ binary combination $(100+100 \mathrm{mg})$ was injected, at controlled flow rate $(15 \mathrm{~mL} / \mathrm{min})$, into $50 \mathrm{~mL}$ of $0.01 \%(\mathrm{w} / \mathrm{v})$ chitosan aqueous solution, under constant mechanical stirring at 15,000 rpm for 10 minutes (Ultraturrax TP 18/10-10N; IKA-Werke GmbH, Staufen, Germany). The $0.01 \%$ chitosan aqueous solution was prepared from a $1 \%$ chitosan solution in $0.03 \mathrm{~N} \mathrm{HCl}$. Volume of organic phase and rate flow injection $(15 \mathrm{~mL} / \mathrm{min})$ were controlled using a mechanical syringe pump coupled with a glass pipette (Model 200; KD Scientific, Holliston, MA, USA). Finally, ethanol phase was evaporated from the prepared colloidal suspension using a rotary evaporator (Heidolph WB/VV 2000; Schwabach, Germany) at the temperature of $40^{\circ} \mathrm{C}$. Batches of LCNs loaded with simvastatin, without oil (SVT-LCNs) and with different oils (SVT-LCN_MaiLab, SVT-LCN_MaiCap SVT-LCN_LabCap , and SVT-LCN_Cap Cap $_{\text {II }}$ ), were produced in order to optimize the formulation for stability and simvastatin encapsulation. All batches were prepared in at least triplicate and stored at room temperature for up to 3 months.

\section{Physicochemical characterization of SVT-LCNs}

Determination of nanoparticle size and surface charge

The particle size and polydispersity index (PDI) of all nanoparticles were determined by dynamic light scattering (DLS) using Malvern Zetasizer Nano ZSP (Malvern Instruments Ltd., Malvern, UK).

For DLS measurements, the colloidal nanoparticle suspensions were diluted with distilled water filtered at $0.45 \mu \mathrm{m}$ to avoid multiple scattering. The analysis was performed at $25^{\circ} \mathrm{C}$ and at a $90^{\circ}$ scattering angle. Three measurements were performed for each sample, in triplicate $(\mathrm{n}=9 \pm$ standard deviation $[\mathrm{SD}])$. 
The surface charge of the nanoparticles was measured using phase analysis light scattering. The same samples were used for both size and zeta potential determination, with the same instrument. For each measurement, nanoparticles were diluted (1:3) with distilled water filtered at $0.45 \mu \mathrm{m}$ to achieve a desired conductance $(300 \mu \mathrm{S})$ without altering the surface charge properties of nanoparticles. Zeta potential values were presented as means of triplicate runs (six subruns) per sample ( $\mathrm{n}=9 \pm \mathrm{SD})$.

\section{Nanoparticle tracking analysis}

In order to further confirm the particle size distribution and have an evaluation of particle concentration in suspension, a nanoparticles tracking analysis (NTA) experiment was conducted using NanoSight NS300 (Malvern Instruments Ltd.) equipped with a $480 \mathrm{~nm}$ laser light source, and a $20 \times$ magnification microscope was used to carry out the particle tracking analysis with a field of view of approximately $100 \times 80 \times 10 \mu \mathrm{m}$. The built-in sCMOS camera was used to record videos, and the particle tracking was analyzed by NTA 3.1 software. NTA tracks single particles in Brownian motion through the light they scatter. Videos of the particle's tracks, projected on the $x-y$ plane, observed through a $20 \times$ microscope, were analyzed by the built-in NTA 3.1 software that locates and follows the center of each individual particle moving in the observation volume, determining the average distance moved by each particle in the $x$ and $y$ directions. This value is then converted into particle size on the basis of a variation of Stokes-Einstein equation taking into account that the motion is tracked in two dimensions:

$$
\overline{(x, y)^{2}}=\frac{4 T k_{\mathrm{B}}}{3 \pi \eta d_{\mathrm{h}}}
$$

where $k_{\mathrm{B}}$ is the Boltzmann constant and $\overline{(x, y)^{2}}$ is the meansquared displacement of a particle during time $t$ at temperature $T$, in a medium of viscosity $\eta$, with a hydrodynamic diameter of $d_{\mathrm{h}}{ }^{34}$

Furthermore, knowing the volume of the suspension and the dilution, the associated NTA software is capable of calculating an approximate concentration of the nanoparticles inside the colloidal suspension. ${ }^{35}$

Only SVT-LCN_MaiLab were analyzed by NTA. The nanoemulsion was highly diluted $(1: 630,000)$ with ultrapure water to allow single particle tracking. After that, sample was drawn into a $1 \mathrm{~mL}$ plastic syringe, which was used for full-sample injection into the instrument sample chamber. The nanoparticle images were acquired using a video capture mode of the sample for three 60-second analyses, which were used for subsequent analysis. Measurement was carried out at a defined temperature $\left(28^{\circ} \mathrm{C}-28.2^{\circ} \mathrm{C}\right)$ and viscosity $(0.828-0.832 \mathrm{cP})$. The results were obtained as mean and SD of three runs.

\section{Nanoparticle imaging by scanning transmission electron} microscopy

The morphology of simvastatin raw material, simvastatinloaded lecithin/chitosan nanospheres (without oily core), blank LCN_MaiLab nanocapsules and SVT-LCN_MaiLab nanocapsules, was observed by scanning transmission electron microscopy (STEM) using an $\mathrm{EVO}^{\circledR}$ electron microscope (ZEISS International, Oberkochen, Germany) operating at an accelerating voltage of $30 \mathrm{kV}$. A drop of sample solution was placed onto a 200 mesh copper grid coated with carbon (Agar Scientific, Stansted, UK) and air dried for 1 minute, after which excess solution was removed gently with filter paper. Subsequently, a drop of $2 \%(\mathrm{w} / \mathrm{v})$ phosphotungstic acid was used as a staining agent and removed after 30 seconds. The images were obtained via inverse contrast imaging with magnification between $75,000 \times$ and $150,000 \times$.

\section{Determination of nanoparticle structure and interaction} with a nasal mucus model by SAXS

The internal structure of nanoparticles and the structural changes occurring upon their interaction with a model of nasal mucus were investigated by Synchrotron small-angle $\mathrm{X}$-ray scattering (SAXS) technique. Experiments were performed at the ID02 high-brilliance beamline (ESRF, Grenoble, France). The X-ray beam cross section was $200 \times 400 \mu \mathrm{m}$ with $\lambda=0.1 \mathrm{~nm}$. All measurements were performed at $T=25^{\circ} \mathrm{C}$. Samples were put in plastic capillaries (KI-BEAM; ENKI srl, Concesio, Italy) with $2 \mathrm{~mm}$ internal diameter, mounted horizontally onto a thermostated sample holder. The region of investigated momentum transfer, $q=(4 \pi / \lambda) \sin (\theta)$, was $0.0116<q<6.43 \mathrm{~nm}^{-1}$, where $2 \theta$ is the scattering angle. In order to prevent any radiation damage, several frames with very short exposure time ( 0.1 second) were acquired, and then checked and averaged. After solvent subtraction, the measured SAXS profiles reported the nanoparticles' scattered radiation intensity as a function of the momentum transfer, $q$. SVT-LCN_MaiLab were prepared according to the above-described protocol at a final concentration of $7.1 \mathrm{mg} / \mathrm{mL}$. In order to assess the stability of SVT-LCN_MaiLab in the presence of artificial mucus and their interaction with lysozyme, SAXS analyses were also 
performed on nanoparticle dispersions in simulated nasal fluid $(8.77 \mathrm{mg} / \mathrm{mL}$ sodium chloride, $2.98 \mathrm{mg} / \mathrm{mL}$ potassium chloride, and $0.59 \mathrm{mg} / \mathrm{mL}$ calcium chloride dihydrate) at three different mucus concentrations $2 \%, 1 \%$, and $0.5 \%$ $(\mathrm{w} / \mathrm{v})$, and the kinetics of interaction in the presence of both mucus $(0.5 \%)$ and lysozyme $(0.5 \mathrm{mg} / \mathrm{mL})$ was followed within 8 hours.

\section{Quantification of simvastatin in LCNs}

The simvastatin content in nanoparticles was measured using a high-performance liquid chromatography (HPLC) system. The instrumentation consisted of ESA model 542 autosampler (Chelmsford, MA, USA), ESA model 584 pump and Shimadzu SPD10A UV detector (Kyoto, Japan). A reverse phase $\mathrm{C}_{18}$ column (Luna, $250 \times 3.0 \mathrm{~mm}, 5 \mu \mathrm{m}$; Phenomenex, Torrance, CA, USA) was employed for chromatographic separation of both simvastatin's isoforms, that is, lactone and hydroxy-acid form, ${ }^{36}$ with a mobile phase consisting of a $65: 35(\mathrm{v} / \mathrm{v})$ mixture of acetonitrile and $0.025 \mathrm{M}$ sodium dihydrogen phosphate buffer $(\mathrm{pH} 4.5)$ at a flow rate of $0.8 \mathrm{~mL} / \mathrm{min}$. The UV detector was set at $238 \mathrm{~nm}$ with a sample injection volume of $50 \mu \mathrm{L}$. In order to quantitatively convert simvastatin into its hydroxy-acid form, simvastatin was dissolved in ethanol and added to 1.5 volumes of $1 \mathrm{~N} \mathrm{NaOH}$ and heated at $50^{\circ} \mathrm{C}$ for 2 hours. Next, the $\mathrm{pH}$ of the solution was adjusted to 7.2 with $\mathrm{HCl}$, and the volume was made up with water to $10 \mathrm{~mL}$. Linearity of calibration curves for both simvastatin and its hydroxy-acid form was verified in the range of $0.5-50 \mu \mathrm{g} / \mathrm{mL}$ ( $r=0.998$ and $r=0.999$, respectively). Limit of detection and limit of quantification were 0.02 and $0.08 \mu \mathrm{g} / \mathrm{mL}$ for simvastatin and 0.06 and $0.18 \mu \mathrm{g} / \mathrm{mL}$ for simvastatin hydroxy-acid form, respectively.

The encapsulation efficiency (EE) of nanoparticles was determined by an indirect method; that is, the amount of precipitated and free simvastatin were quantified and subtracted from the total amount of the drug quantified in the total preparation, and all amounts were determined by HPLC, and then expressed as a percentage of the total drug in the preparation. The total amount of simvastatin in the formulations was quantified. Firstly, $100 \mu \mathrm{L}$ of the preparation was dispersed in $10 \mathrm{~mL}$ of ethanol by sonication for 15 minutes (ultrasonic cleaner; VWR, Radnor, PA, USA) to extract the entire amount of drug. Then, the ethanol dispersion was heated at $60^{\circ} \mathrm{C}$ in a tightly closed container for 5 minutes to solubilize the drug content extracted from the nanoparticles and directly assayed for HPLC. For the quantification of precipitated and free simvastatin in the aqueous medium, colloidal suspensions were first centrifuged at $1,500 \times \mathrm{g}$ for
10 minutes (Medifuge; Heraeus Sepatech Gmbh, Hanau, Germany) to separate the nanoparticles from any large precipitate. The pellet obtained for each sample was resuspended with ethanol, submitted to a brief sonication process to solubilize the drug and then analyzed by HPLC. An aliquot of $2 \mathrm{~mL}$ of supernatant obtained in the previous step was further centrifuged using Vivaspin ${ }^{\circledR}$ Centrifugal Concentrator (MWCO $30,000 \mathrm{Da}$; Sartorius) at 4,000 $\times \mathrm{g}$ for 10 minutes (Medifuge, Heraeus Sepatech Gmbh) to separate the dissolved and hence non-encapsulated simvastatin. The ultrafiltered solution was diluted with ethanol by the same method used for pellet quantification and analyzed by HPLC as well. All analyses were performed in triplicate $(n=9 \pm S D)$. EE of simvastatin was determined by using the following formula:

$$
\mathrm{EE} \%=\frac{\begin{array}{l}
\text { Total amount of SVT }- \\
(\text { Precipitated SVT }+ \text { Dissolved SVT })
\end{array}}{\text { Total amount of SVT }} * 100
$$

In vitro drug release from $\mathrm{LCNs}$

In vitro release from SVT-LCN_MaiLab nanocapsules was studied using simulated nasal electrolytic solution (SNES) containing potassium, calcium, and sodium at biologic human concentrations of the nasal fluid, as described by Castile et al. ${ }^{37}$ In order to obtain sink conditions during the release experiments, simvastatin solubility in SNES was increased by adding $0.5 \%(\mathrm{w} / \mathrm{v}) \mathrm{BSA}$ to the dissolution medium.

The test for in vitro release from SVT-LCN_MaiLab in the SNES- $0.5 \%$ BSA release medium at $\mathrm{pH} 6.5$ was conducted using the dialysis bag technique (dialysis tube membrane, MWCO 14,000 kDa; Sigma-Aldrich). A volume of $1 \mathrm{~mL}$ of the nanoemulsion was diluted with $1 \mathrm{~mL}$ of SNES at pH 6.5 and placed in the dialysis bags. The sealed bags were immersed into $100 \mathrm{~mL}$ of the release medium containing $0.5 \% \mathrm{BSA}$ kept at $37^{\circ} \mathrm{C}$ and magnetically stirred at $100 \mathrm{rpm}$. At predetermined time points $(0,1,2,3,4,5,6,7,8$, and 24 hours), $1.5 \mathrm{~mL}$ aliquots of the dissolution medium were taken and replaced with an equivalent amount of fresh release medium. Samples were analyzed by HPLC to determine the released simvastatin. The same experiment was conducted using a $1 \mathrm{mg} / \mathrm{mL}$ simvastatin suspension in ultrapure water used as control. In vitro release studies were replicated three times for both simvastatin nanoparticles and simvastatin suspension.

\section{Cytotoxicity assay of SVT-LCNs}

Cytotoxicity assays of simvastatin suspension, SVT-LCN MaiLab and Blank-LCN_MaiLab were conducted using the human nasal septum carcinoma cell line RPMI 2650 (ATCC) 
and performing an MTT [3-(4,5-dimethylthiazol-2-yl)2,5-diphenyltetrazolium bromide] cytotoxicity assay. The RPMI 2650 cell line was cultured in an MEM containing 10\% (v/v) FBS and 1\% nonessential amino acid solution and incubated at $37^{\circ} \mathrm{C}$ with $95 \%$ air humidity and $5 \% \mathrm{CO}_{2}$ atmosphere. Cells were seeded at an initial density of 50,000 cells per well and incubated for 24 hours to allow cell adhesion in 96-well cell culture cluster (Costar; Corning Incorporated). Different concentrations from 0 to $240 \mu \mathrm{M}$ of simvastatin nanoparticles, SVT-LCN_MaiLab and Blank-LCN_MaiLab were prepared by direct dilution in cell culture medium. To prepare the raw simvastatin solution, dimethyl sulfoxide (DMSO) was used as simvastatin solvent and diluted to low final concentration $(<0.1 \%)$ to avoid toxicity effects of the solvent on the cell viability. The cells were treated with the formulations for 72 hours, followed by incubation in MTT reagent for 2 hours at $37^{\circ} \mathrm{C}$. Next, the cell medium was removed, and $120 \mu \mathrm{L}$ of DMSO was added to each well to dissolve the violet-colored metabolite. The plates were shaken for 15 minutes, the contents were pipetted and transferred to new plates and the absorbance was measured at $570 \mathrm{~nm}$ using a microplate reader (Spark 10 M; Tecan, Männedorf, Switzerland). Absorbance values were considered directly proportional to cell viability, and percentage cell viability was calculated by comparison to control values obtained for untreated cells.

\section{Gamma scintigraphy studies}

Particles labeling with ${ }^{99 m} T c$

A preliminary experiment to evaluate the administration of simvastatin-loaded nanoparticles was carried out in rats by gamma scintigraphy. SVT-LCN_MaiLab and a simvastatin suspension were both labeled with ${ }^{99 \mathrm{~m}} \mathrm{Tc}$ based on previous experiences, in which anti-CD3 monoclonal antibody was successfully labeled. ${ }^{38-40}$

Briefly, $60 \mu \mathrm{L}$ of simvastatin nanoparticles or simvastatin suspension $(1 \mathrm{mg} / \mathrm{mL})$ was incubated with $100 \mu \mathrm{L}$ of $\mathrm{SnCl}_{2}(0.6,6$, or $60 \mu \mathrm{g})$ (Sigma-Aldrich) in $0.9 \% \mathrm{NaCl}$ for 20 minutes at room temperature. Afterward, $100 \mu \mathrm{L}(100 \mu \mathrm{Ci})$ of pertechnetate ( ${ }^{99 \mathrm{~m}} \mathrm{TcO}^{4-}$; CNEN/IPEN, São Paulo, Brazil) was added, and the reaction mixture was incubated for further 10 minutes at room temperature. The radiolabeling efficiency of the nanoparticle and the simvastatin was evaluated using thin-layer chromatography (TLC), which was carried out using Whatman filter paper No 1 and acetone as mobile phase. The radioactivity of the strips was quantified in a gamma counter (Wizard2; PerkinElmer, Waltham, MA, USA). The nanoparticle and the simvastatin were both successfully labeled using $6 \mu \mathrm{g}$ of $\mathrm{SnCl}_{2}$ reaching an average $94 \%$ labeling efficiency. To quantify the TLC results, the distance traveled by the substance being considered is divided by the total distance traveled by the mobile phase. This is called the retention factor ( $\mathrm{Rf})$; in this experiment, free ${ }^{99 \mathrm{~m}} \mathrm{Tc}$ pertechnetate had a high Rf being transported with the mobile phase. When the particle labeling was carried out, the new radiopharmaceutical conjugate was not efficiently eluted by the mobile phase; that is, $\mathrm{Rf}=0$. Efficient radiolabeling was considered for a signal at $\mathrm{Rf}=0 \%>80 \%$.

\section{In vivo biodistribution study}

All animal experiments were approved by the Ethics in Research Committee of University Hospital Clementino Fraga Filho (affiliated university Federal University of Rio de Janeiro, Rio de Janeiro, Brazil; [CECA/CCS/UFRJ 129/14]). All animals were handled in accordance with Brazilian guidelines for the care and use of animals for scientific and educational purposes (Conselho Nacional de Controle de Experimentação Animal - CONCEA, 2016). Nine Wistar rats weighing 300-350 g obtained from the central vivarium were used. The animals had free access to a standard rat diet and tap water at all times during the studies. Three Wistar rats per group were placed individually in an induction chamber, and anesthesia was induced with $1 \%$ isoflurane. Then, $10 \mu \mathrm{L}$ of the radiolabeled formulation, that is, SVT-LCN_MaiLab, simvastatin suspension or a simple pertechnetate solution $\left(\mathrm{TcO}^{4-}\right)$, was instilled with a micropipette in each nostril of the animal. Ninety minutes post-administration, the animals were sacrificed with a high dose of isoflurane (5\%), and the organs were collected for biodistribution analysis.

For quantitative biodistribution analysis, the brain, heart, lungs, liver, kidneys, spleen, and stomach of rats were removed and weighed. The total radioactivity administered to each animal and the radioactivity present in each organ were measured in a gamma counter (Packard Cobra II AutoGamma Counter; PerkinElmer). The percentage of the dose per gram of the organ (\% gram/tissue: \% dose/organ/mass in grams) was determined for each sample.

\section{Statistics}

All results were reported as mean and SD of at least three replicates, unless stated otherwise. Cytotoxicity $\mathrm{IC}_{50}$ values were calculated by using a nonlinear (sigmoidal, 4PL) fitting of each data set (Prism, Version 7.0a; GraphPad Software Inc., La Jolla, CA, USA). The differences between data were tested using Student's $t$-test (paired, two-tailed) considering significant differences with $P<0.05$. The results of the biodistribution assays were analyzed using two-way analysis of variance, and a Tukey's multiple comparisons test was used to compare the groups. Differences were considered to 
be statistically significant at $P<0.001$ (Prism, Version 7.0a; GraphPad Software Inc.).

\section{Results}

\section{Preparation and physicochemical characterization of SVT-LCNs}

Simvastatin nanoparticles were formed via the electrostatic self-assembly of lecithin and chitosan. In previous papers, this system was shown to be adapted for the encapsulation of lipophilic drugs, but the loading efficiency was limited by the affinity of the drug for the phospholipid component of the nanosystem. ${ }^{31,41}$

In this study, in order to improve their loading capacity, nanoparticles were produced by adding to the formulation different oils on the basis of literature solubility studies of simvastatin. Maisine, Labrafac, Capryol PGMC, and Capryol 90 were chosen, and formulated with lecithin/chitosan in binary combinations, at the maximum amount compatible with the production of stable nanoparticles. Preliminary experiments were carried out to determine the maximum amount of oils that could be used to produce stable nanoparticles (data not shown).

To select the optimal formulation, all nanoparticles prepared were characterized for size, PDI, zeta potential, drug-loading efficiency, and stability during 3-month storage at room temperature. Table 1 reports the average size, PDI, zeta potential, and drug-loading efficiency of SVT-LCNs produced without oil and with different oil combinations.

SVT-LCNs produced without oily excipients showed higher average size (around $270 \mathrm{~nm}$ ) and smaller positive surface charge compared to blank nanoparticles (LCNs). Furthermore, substantial precipitation of simvastatin affected the EE of SVT-LCN. The EE was approximately $22 \%$. In all the examined cases, the addition of oil significantly reduced the amount of drug precipitated during preparation of nanoparticles. This was consistent with an improved loading capacity provided by adding an oily core to nanoparticles, leading to a fourfold increase in EE. In preliminary studies carried out with only Maisine, it was observed that the EE of simvastatin was increasing with increasing oil concentration (Table 1 presents the data for LCN_Mai and LCN_Mai ${ }_{2}$ ). It was hypothesized that the combination of different types of oils could promote a further improvement in EE. In most cases, no significant improvement was obtained. It can be seen in Table 1 that the addition of the oil combinations $\mathrm{Cap}_{\mathrm{I}} \mathrm{Cap}_{\text {II }}$ and LabCap in formulations (SVT-LCN_Cap I Cap and SVT-LCN_LabCap ) did not result in a significant variation in the average size compared to SVT-LCNs or EE when compared to lecithin/chitosan Maisine-containing nanoparticles (SVT-LCN_Mai ${ }_{2}$ ). On the other hand, SVTLCN_MaiCap I showed larger particle size (352 nm) but not a dramatic increase in loading capacity. All the oil-containing formulations evidenced a positive surface charge with values ranging from +11 to $+48 \mathrm{mV}$. The preparations with higher particle sizes also showed reduced surface charge and higher PDI, with a potential negative effect on their long-term stability. In fact, SVT-LCN_Cap ${ }_{I} \mathrm{Cap}_{\text {II }}$ and SVTLCN_MaiCap I preparations evidenced precipitation or flocculation just few days after preparation. SVT-LCN_LabCap was apparently stable over 1-month storage but displayed a slight phase separation over longer times.

On the other hand, the addition of the oil combination Maisine and Labrafac had a huge positive effect. In fact, simvastatin-loaded nanoparticles produced using Maisine and Labrafac (SVT-LCN_MaiLab) showed a significantly smaller particle size $(204 \mathrm{~nm})$, elevated positive surface charge (nearly $50 \mathrm{mV}$ ) and high drug-loading efficiency, encapsulating $98 \%$ of the total amount of simvastatin. Moreover, SVT-LCN_MaiLab were found to be monodispersed (PDI $<0.1$ ). In addition, the system remained chemically and physically stable at room temperature up to 3 months, as shown in Table 2. Therefore, the SVT-LCN_MaiLab

Table I Physicochemical properties and EE of simvastatin-loaded nanoparticles ( $n=9 \pm S D)$

\begin{tabular}{|c|c|c|c|c|c|}
\hline Formulation & Oil ratio & Particle size $(\mathrm{nm})$ & Zeta potential $(\mathrm{mV})$ & PDI & EE (\%) \\
\hline LCN & - & $146.7 \pm 26.2$ & $+57.26 \pm 2.57$ & $0.380 \pm 0.025$ & - \\
\hline SVT-LCN & - & $272.0 \pm 12.6$ & $+34.43 \pm 2.13$ & $0.263 \pm 0.040$ & $22.60 \pm 20.80$ \\
\hline SVT-LCN_Mai & $\mathrm{I}: 0$ & $192.4 \pm 22.5$ & $+36.63 \pm 2.25$ & $0.120 \pm 0.035$ & $74.13 \pm 6.04$ \\
\hline SVT-LCN_Mai & $2: 0$ & $184.9 \pm 9.5$ & $+45.30 \pm 1.08$ & $0.089 \pm 0.004$ & $86.90 \pm 6.28$ \\
\hline LCN_MaiLab & $\mathrm{I}: \mathrm{I}$ & $205.6 \pm 10.2$ & $+50.20 \pm 2.17$ & $0.129 \pm 0.017$ & - \\
\hline SVT-LCN_MaiLab & I:I & $204.5 \pm 15.4$ & $+48.45 \pm 4.09$ & $0.098 \pm 0.040$ & $98.52 \pm 1.33$ \\
\hline SVT-LCN_Cap,Cap & $\mathrm{I}: \mathrm{I}$ & $280.4 \pm 9.2$ & $+20.17 \pm 2.03$ & $0.185 \pm 0.045$ & $88.78 \pm 0.99$ \\
\hline SVT-LCN_MaiCap, & $\mathrm{I}: \mathrm{I}$ & $352.0 \pm 33.9$ & $+\mid 1.34 \pm 3.21$ & $0.223 \pm 0.020$ & $90.74 \pm 0.87$ \\
\hline SVT-LCN_LabCap, & $1: 1$ & $27 I .1 \pm 8.1$ & $+|7.0| \pm 2.98$ & $0.154 \pm 0.040$ & $94.68 \pm 0.65$ \\
\hline
\end{tabular}

Note: '-' indicates no data.

Abbreviations: EE, encapsulation efficiency; SD, standard deviation; PDI, polydispersity index; LCN, lecithin/chitosan nanoparticle; SVT-LCN, simvastatin-loaded lecithin/ chitosan nanoparticle; Mai, Maisine; Mai ${ }_{2}$, double Maisine; Lab, Labrafac; Cap, propylene glycol monocaprylate type I; Cap», propylene glycol monocaprylate type II. 
Table 2 Physical and chemical stability study at room temperature of simvastatin-loaded nanoparticles (SVT-LCN_MaiLab)

\begin{tabular}{lllll}
\hline Storage time (months) & Particle size $(\mathbf{n m})$ & Zeta potential $(\mathbf{m V})$ & PDI & EE (\%) \\
\hline 0 & $204.5 \pm 15.4$ & $48.4 \pm 4.1$ & $0.098 \pm 0.040$ & $98.52 \pm 1.33$ \\
I & $205.5 \pm 15.2$ & $48.1 \pm 3.2$ & $0.166 \pm 0.024$ & $97.11 \pm 1.25$ \\
3 & $201.9 \pm 18.6$ & $40.0 \pm 2.5$ & $0.131 \pm 0.033$ & $96.54 \pm 1.13$ \\
\hline
\end{tabular}

Abbreviations: PDI, polydispersity index; EE, encapsulation efficiency; SVT-LCN, simvastatin-loaded lecithin/chitosan nanoparticle; Mai, Maisine; Lab, Labrafac.

formulation was selected for further experiments, as it presented small and narrow particle size distribution, positive and sufficiently high superficial charge and optimal drug entrapment efficiency.

\section{Nanoparticle tracking analysis}

In order to confirm the particle-sizing data obtained by DLS, SVT-LCN_MaiLab particle size distribution and concentration were measured using NTA. NTA is a relatively new investigation technique that offers direct and real-time visualization, sizing, and counting of nanoparticles, allowing high-resolution particle size distributions to be obtained. ${ }^{42}$ Results are shown in Figure 1. The particle size distribution showed a peak at $101.0 \pm 4.6 \mathrm{~nm}$ with $90 \%$ of the particles being $<132.1 \pm 13.0 \mathrm{~nm}$, confirming the narrow size distribution of the nanoparticles. A smaller NTA average particle size in comparison with DLS results was expected due to the different weighting functions and the intensity scattered by particles for DLS which is much larger for large particles. NTA provides complementary information to both DLS and microscopy. In fact, as it follows individual particles, it enhances the resolution of polydispersed particle population

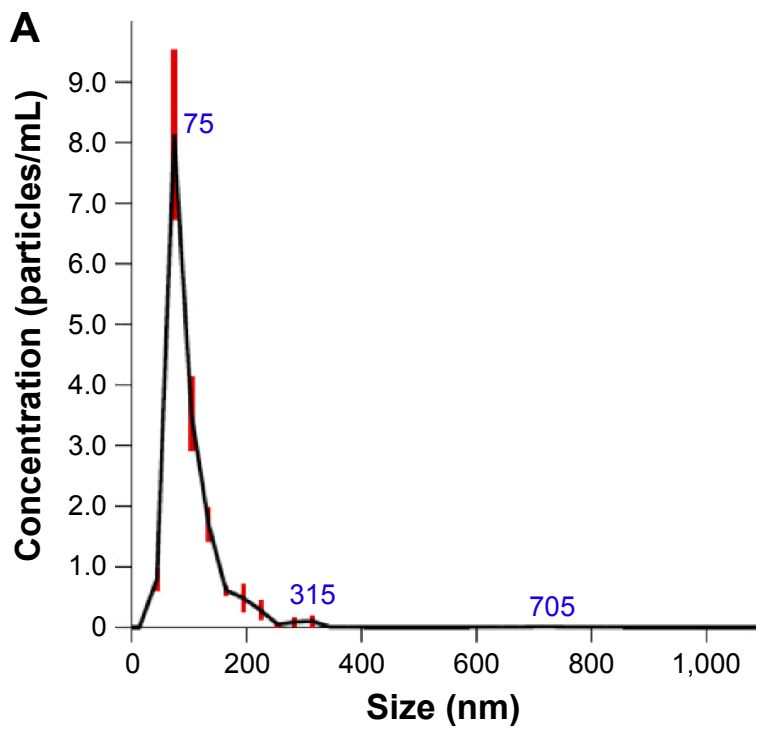

which is usually obtained by DLS. The technique still operates on a statistically significant number of particles, larger than for microscopy, although not determining their morphology. ${ }^{43}$

NTA measurements also confirmed the stability of the lecithin/chitosan oil core nanoparticles prepared using Maisine and Labrafac combination, as similar results were obtained for the same formulation stored for 3 months at room temperature (data not shown).

\section{Nanoparticles imaging by STEM}

LCNs were further characterized by STEM (Figure 2). Simvastatin raw material, SVT-LCNs and SVT-LCNs prepared using Maisine and Labrafac (SVT-LCN_MaiLab) are shown in Figure 2A-C, respectively. Simvastatin suspension was organized as agglomerates of individual elongated crystals of few micrometers in size (Figure 2A). LCNs prepared without oil loaded with simvastatin produced spherical spongy particles in the range of $100-500 \mathrm{~nm}$, as shown in Figure 2B. Large simvastatin crystals were also observed within this formulation, as a consequence of low encapsulation efficacy, and consistently showed extensive precipitation during nanoparticle preparation. The incorporation of oils, that is, Maisine

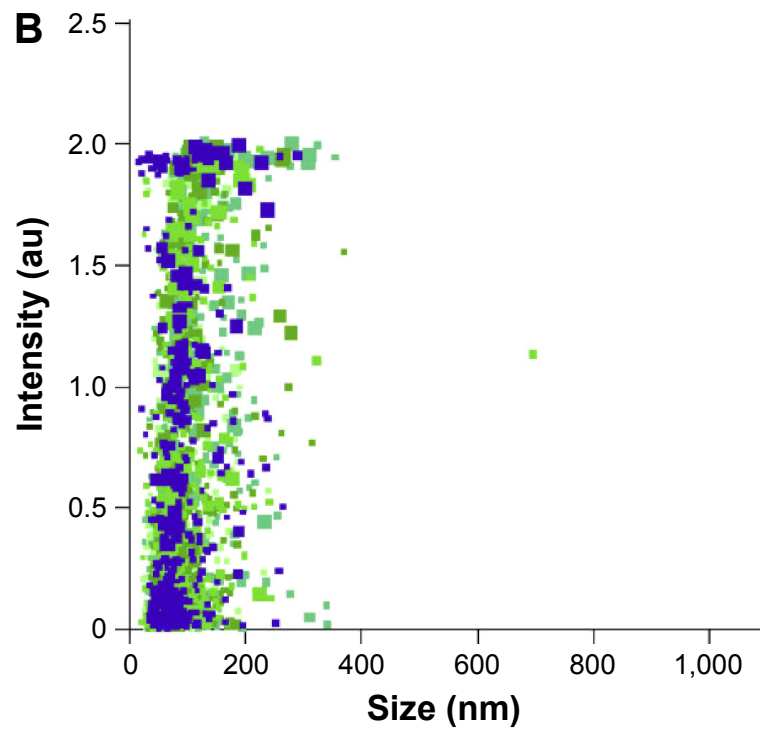

Figure I Particle size distribution vs nanoparticles concentration and intensity of scattered light obtained by NTA.

Notes: Particle size distribution is expressed as average and standard error of the mean of nanoparticle concentration ( $n=5)(\mathbf{A})$. Different colors and sizes of markers represent measures of particle size and scattered light intensity of single particles from the five independent experiments (B).

Abbreviation: NTA, nanoparticles tracking analysis. 

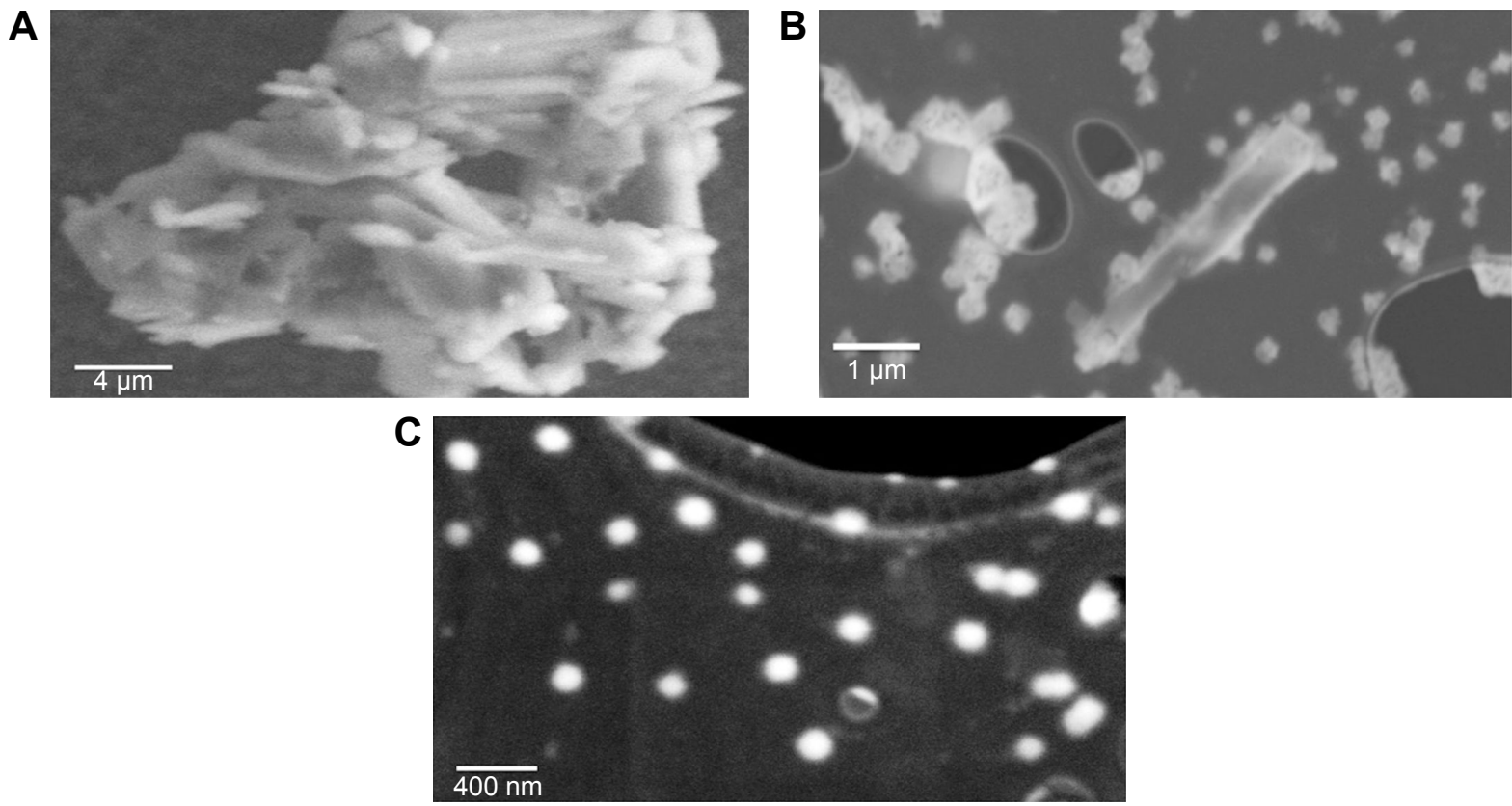

Figure 2 STEM images of simvastatin crystals (A), SVT-LCNs (B), and SVT-LCN_MaiLab (C).

Abbreviations: STEM, scanning transmission electron microscopy; SVT-LCNs, simvastatin-loaded lecithin/chitosan nanoparticles; Mai, Maisine; Lab, Labrafac.

and Labrafac, into the formulation of the LCNs significantly improved the nanoparticle morphology and size distribution. SVT-LCN_MaiLab (Figure 2C) appeared as small almost perfectly spherical nanoparticles with narrow size distribution $(150-250 \mathrm{~nm})$. Additionally, the increased EE of simvastatin following incorporation of oils into the formulation was confirmed by the absence of large simvastatin crystals.

\section{Nanoparticles structure and interaction with the nasal mucus model}

To investigate the internal structure of nanoparticles, SAXS measurements were performed on blank and simvastatin-loaded nanoparticles. In Figure 3, we report the scattered X-ray intensity profiles for LCN, LCN_MaiLab, and SVT-LCN_MaiLab.

Spectra were very different all over the investigated $q$ region. Blank LCNs (bottom black line in Figure 3) showed the characteristic features of closed lamellar structures, such as vesicles, with low multilamellar layering as evidenced previously. ${ }^{30}$ The structure peak at $q=1.13 \mathrm{~nm}^{-1}$ corresponded to a characteristic interlamellar distance of $5.6 \mathrm{~nm}$. In fact, the momentum transfer $q$ was related to the characteristic scattering distance of supramolecular structures $d$ by the equation:

$$
d=\frac{2 \pi}{q}
$$

The obtained bilayer contrast profile is reported in the insert of Figure 3 (bottom black profile). The structural parameters were in agreement with typical values for lipid/polysaccharide nanoparticles: ${ }^{30}$ the overall size was $140 \pm 20 \mathrm{~nm}$, and the hollow water core was surrounded by one or more lipid bilayers, each $5 \mathrm{~nm}$ thick, with the interlayer regions containing water and chitosan.

Intensity spectra of LCN systems containing Maisine and Labrafac oils (LCN_MaiLab, blue line in Figure 3) clearly revealed the presence of an oil core that definitely changed the

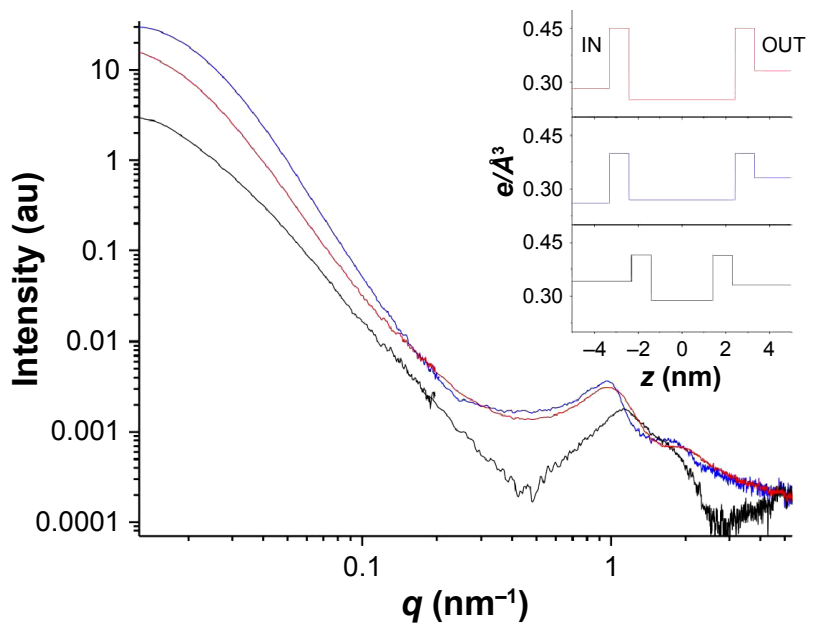

Figure 3 SAXS intensity spectra of LCN (black line), LCN_MaiLab (blue line), and SVT-LCN_MaiLab (red line).

Notes: The corresponding electron density profiles $\left(\mathrm{e} / \AA^{3}\right)$ across the bilayer are shown in the insert. IN is the core region, and OUT is the bulk solvent region and $z$ is the distance from the center of the bilayer. $\left(e / \AA^{3}\right)$ is the electron density for number of electrons/ volume.

Abbreviations: SAXS, small-angle X-ray scattering; LCNs, lecithin/chitosan nanoparticles; Mai, Maisine; Lab, Labrafac; q, momentum transfer. 
contrast of the inner region, the "IN" electron density being lower than the "OUT" (insert of Figure 3, central blue profile). The intensity decay in the low- $q$ region was proportional to $q^{-4}$, as expected for globular particles with well-defined interfaces. The overall size of oil-containing particles was roughly $200 \mathrm{~nm}$. The nanoparticle structure was core-shell type: the oil core was surrounded by a multilayer shell, as visible in the high- $q$ region of the spectra. The obtained profile of bilayers belonging to the shell showed that their structure was affected by the presence of the oil component, as reported in Figure 3. The internal hydrophobic region of the lamellae was thicker than for blank nanoparticles. Accordingly, the interlamellar peak appeared at lower $q$ values $\left(1 \mathrm{~nm}^{-1}\right)$ corresponding to a characteristic distance of $6.3 \mathrm{~nm}$, larger than for oil-free nanoparticles. Simvastatin-loaded nanoparticles (SVT-LCN_ MaiLab) showed a structure quite similar to the unloaded ones. Interestingly, simvastatin seemed not only to be embedded in the oil core, but also in the shell structure, lowering its electron density as compared to the unloaded nanoparticles.

To investigate the structural changes induced by the interaction of the nanoparticles with mucus, SAXS analyses were also performed on SVT-LCN_MaiLab dispersions in the presence of artificial mucus in simulated nasal fluid $(2 \%, 1 \%, 0.5 \%$ $[\mathrm{w} / \mathrm{v}])$. Figure 4, left panel, reports the intensity spectrum of the nanoparticles in $0.5 \%$ artificial mucus together with that of the mucus itself. In the right panel, the spectrum obtained originally for the nanoparticles in simulated nasal fluid is compared to the one of the nanoparticles inside the mucus, after subtraction of the mucus contribution to the scattered intensity.

First, it was observed that in the presence of mucus, nanoparticles were still detected and kept the same core-shell

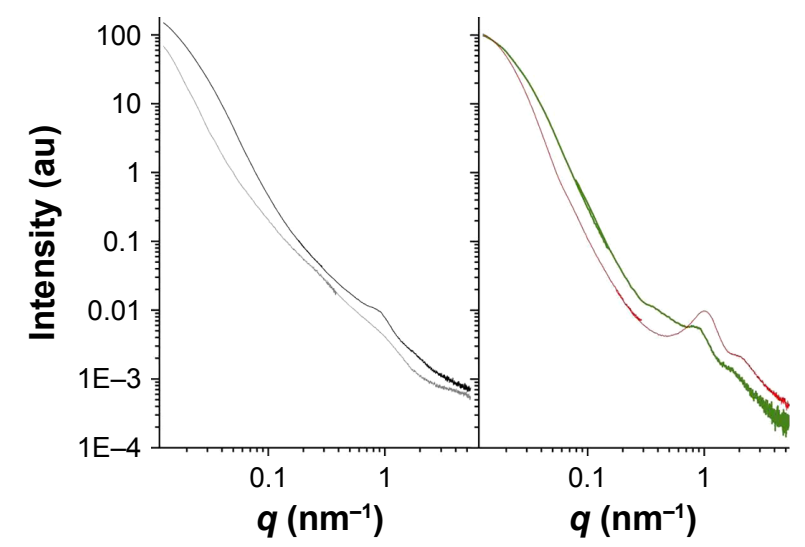

Figure 4 SAXS intensity spectra of SVT-LCN_MaiLab dispersed in artificial mucus. Notes: Left panel: SVT-LCN_MaiLab in $0.5 \%$ artificial mucus (black line) and $0.5 \%$ mucus (gray line). Right panel: SVT-LCN_MaiLab before interaction with artificial mucus (red line) and in mucus, after mucus spectrum subtraction (green line).

Abbreviations: SAXS, small-angle X-ray scattering; q, momentum transfer; SVTLCNs, simvastatin-loaded lecithin/chitosan nanoparticles; Mai, Maisine; Lab, Labrafac. structure. Differences were visible in the high- $q$ region of the spectra, corresponding to the local scale. The characteristic structure peak shifted to $q=0.89 \mathrm{~nm}^{-1}$, corresponding to an interlamellar distance of $7 \mathrm{~nm}$. The adjacent bilayers swelled, as indicated by the increased thickness of the interlamellar solvent layer, showing that the particle was stretched by the presence of the mucus matrix. Meanwhile, the intensity of the structure peak was decreased, suggesting a peeling off of layers from the multilayer shell. Moreover, an additional small peak at $q=0.38 \mathrm{~nm}^{-1}$ stemmed for polymer (chitosan and/or mucin) coordination, with a correlation length of $\sim 16.5 \mathrm{~nm}$.

Finally, the effect and kinetics of the interaction upon addition of lysozyme to the mucus/nanoparticles system were tested, following its structural evolution over several hours. Lysozyme is a protein widely present in natural mucosal secretions, being one of the most abundant antimicrobial factors that constitute the innate immunity. ${ }^{44}$ Lysozyme at a physiological concentration $(0.5 \mathrm{mg} / \mathrm{mL})^{45}$ was added in mucus plus SVT-LCN_MaiLab dispersion, and subsequent SAXS spectra were acquired at different incubation times. The corresponding intensity spectra are reported in Figure 5. Experimental results revealed that lysozyme interacts with nanoparticles helping the peeling process of the multilayer shell. In fact, both the characteristic structure peaks were moving to lower $q$ values, and the associated intensity decreased. After 6 hours, the effect was almost complete. In the first hours, the external

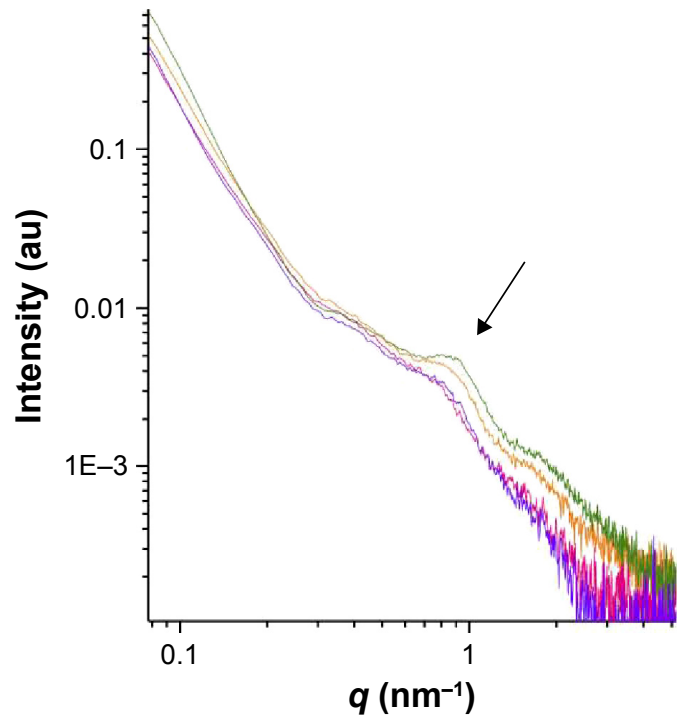

Figure 5 SVT-LCN_MaiLab interaction with mucus plus lysozyme. Notes: SAXS intensity spectra of SVT-LCN_MaiLab in artificial mucus $(0.5 \%)$ after mucus subtraction: without lysozyme (green line) and in interaction with lysozyme at different times: $t=0$ hours (orange line), $t=6$ hours (pink line), and $t=8$ hours (violet line). The arrow indicates the position of the characteristic structure peak of nanoparticles.

Abbreviations: SAXS, small-angle X-ray scattering; SVT-LCNs, simvastatin-loaded lecithin/chitosan nanoparticles; Mai, Maisine; Lab, Labrafac; q, momentum transfer. 
layers of the shell progressively swelled and peeled off suggesting a specific biodegradation of the nanoparticles as a consequence of the interaction with the enzyme.

\section{Simvastatin release studies}

In vitro release testing is an important analytical tool, used to investigate and establish product behavior and stability during each step of the drug development. ${ }^{46}$ The in vitro drug release was measured via the dialysis bag diffusion method which is one of the most commonly used dissolution methods for the testing of nanoformulations designed for nasal drug delivery. ${ }^{47-49}$ SVT-LCN_MaiLab were chosen for testing due to their optimal EE of drug and suitable physicochemical features, and a suspension of simvastatin was used as control. The simvastatin release profile from SVT-LCN_MaiLab can be seen in Figure 6. A simulated nasal fluid at $\mathrm{pH}$ 6.5, containing sodium, potassium, and calcium salts, was used to simulate the nasal conditions. Due to the low solubility of simvastatin in aqueous solution, BSA was used to increase simvastatin solubility in the dissolution medium outside the dialysis bag to achieve sink conditions throughout the experiment. BSA was selected for being closer to physiological conditions in comparison to surfactants or co-solvents generally used to increase the solubility of poorly soluble drugs. The in vitro release tests were performed over 24 hours for SVT-LCN_MaiLab and simvastatin suspension. For the suspension, after an initial rapid release in the first hour, a plateau characterized by a very low dissolution rate was reached. This was not observed for the nanoparticle formulation. In fact, the nanoparticles kept releasing simvastatin at a constant release rate from the second hour to the end of the experiment. As shown in Figure 6, 40\% of simvastatin was released from

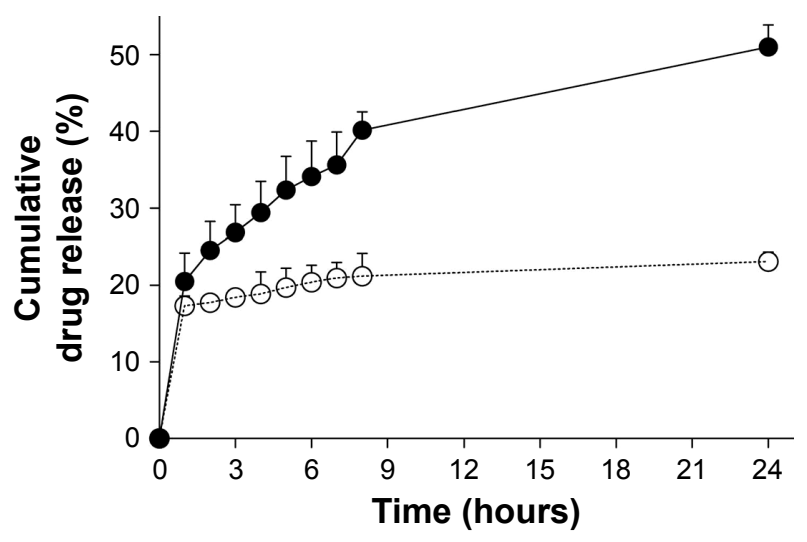

Figure 6 Simvastatin release profile from SVT-LCN_MaiLab (filled circle) and a control simvastatin suspension (open circle) in simulated nasal fluid with $0.5 \%$ BSA at $\mathrm{pH} 6.5$ and $37^{\circ} \mathrm{C}$.

Abbreviations: BSA, bovine serum albumin; SVT-LCNs, simvastatin-loaded lecithin/ chitosan nanoparticles; Mai, Maisine; Lab, Labrafac.
SVT-LCN_MaiLab within 8 hours and $>50 \%$ in 24 hours, displaying a significantly faster release than the simvastatin suspension $(21.17 \%$ simvastatin released after 8 hours by the suspension).

\section{Cytotoxicity studies}

In the last few years, the human RPMI 2650 epidermoid carcinoma cells have been proposed as a suitable model of nasal mucosa for in vitro studies simulating nasal drug transport. ${ }^{50}$ Recently, this nasal epithelial cell line has been grown in air-liquid interface conditions to develop an in vitro model of the nasal mucosa suitable for studies of deposition and permeation of nasally administered formulations. These cells also appear a good choice for in vitro cytotoxicity assay of a new formulation such as SVT-LCNs. For this purpose, RPMI 2650 cells were incubated for 72 hours with increasing concentrations of simvastatin solution, blank nanoparticles, and simvastatin-loaded nanoparticles. The cells viability was recorded as percentage in comparison to untreated cells and plotted in Figure 7 against the simvastatin concentration. In the case of blank nanoparticles, this value corresponded to the equivalent amount of carrier nanoparticles.

Blank particles did not show any cytotoxicity indicating that the carrier is suitable for nasal administration. Results showed that cells viability also remained around $100 \%$ in the highest concentration, suggesting that our LCNs containing oil are highly biocompatible.

Cytotoxicity of simvastatin and SVT-LCN_MaiLab appeared to be dose-dependent, as seen through the cells viability. For SVT-LCN_MaiLab, $\mathrm{IC}_{50}$ was found to be $9.92 \mu \mathrm{M}$, which was nearly three times that of pure simvastatin $(3.50 \mu \mathrm{M})$, and displayed a reduced toxicity compared to the pure drug.

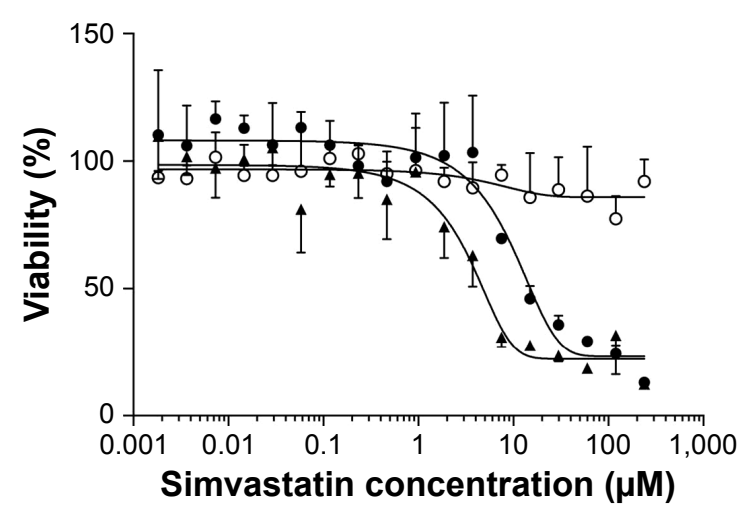

Figure 7 In vitro cytotoxicity studies on human nasal cell line RPMI 2650 of simvastatin (filled triangle), simvastatin-loaded nanoparticles (SVT-LCN_MaiLab, filled circle), and blank nanoparticles (LCN_MaiLab, open circle).

Notes: Cell viability is plotted against the logarithm of simvastatin concentration.

Abbreviations: SVT-LCNs, simvastatin-loaded lecithin/chitosan nanoparticles; Mai, Maisine; Lab, Labrafac. 


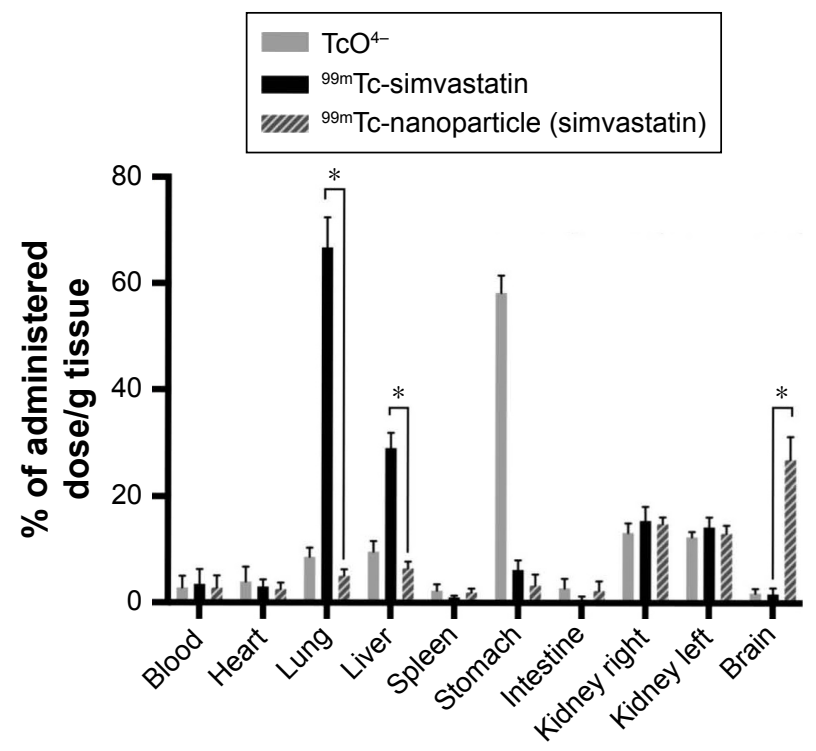

Figure 8 Radioactivity biodistribution in Wistar rats 90 minutes after the nasal instillation of $20 \mu \mathrm{L}\left(10 \mu \mathrm{L}\right.$ in each nostril) of ${ }^{99 \mathrm{~m} T c-l a b e l e d ~ s i m v a s t a t i n-l o a d e d}$ nanoparticles, simvastatin suspension, and pertechnetate $\left(\mathrm{TcO}^{4}\right)$ expressed as percentage of administered dose $(\% D)$ per gram of tissue $(n=3, * P<0.00 I)$.

\section{Gamma scintigraphy studies}

A preliminary study of biodistribution in rats after nasal administration of ${ }^{99 \mathrm{~m}} \mathrm{Tc}$-labeled nanoparticles was carried out by gamma scintigraphy which was compared to the administration of a radiolabeled suspension of simvastatin and pertechnetate solution alone, used as controls. Figure 8 shows organ distribution of the radioactivity detected 90 minutes after the nasal instillation in each nostril of $10 \mu \mathrm{L}$ nanoparticles or drug suspension. The radioactivity distributions were very different. In the case of the drug suspension, most of the radioactivity was found in the lung, followed by far by the stomach and the liver. Only a very limited amount of radioactivity was found in other organs or in the brain. In the case of the pertechnetate solution, radioactivity was mainly found in the stomach. On the contrary, after the nasal administration of nanoparticle formulation, a significant fraction of the radioactivity (more than 20\%) was localized in the brain, followed by an accumulation in the kidneys comparable to the levels observed for controls. Other organs such as the liver, lung, heart, and stomach contained progressively decreasing amounts of radioactivity.

\section{Discussion}

Administration of poorly soluble drugs intranasally for systemic and CNS therapeutic action can be extremely challenging due to the low volume of nasal secretions, the barrier provided by the mucus layer and the short time available for dissolution/absorption because of mucociliary clearance..$^{51,52}$
It is considered that nanoparticles could improve the bioavailability of nasally administered substances.${ }^{53,54}$ Chitosan and lecithin have been used to produce nanoparticles and liposomes for many years. ${ }^{55-57}$ The main advantages attributed to these components are their biocompatibility and biodegradability, and in fact, liposomes are still by far the most successful nanomedicines on the market. ${ }^{58}$ Recently, our research group proposed hybrid nanoparticles formed by the electrostatic interaction of these two components, that is, the positively charged polysaccharide chitosan and the negatively charged phospholipids of soybean lecithin. ${ }^{59,60}$ These nanoparticles were demonstrated to be extremely effective in the improvement of accumulation of corticosteroid drugs in skin layers and of the permeation of tamoxifen through the intestinal epithelium. ${ }^{31,61,62}$

As a consequence, it has been hypothesized that this delivery system could be an interesting candidate for the nasal administration of lipophilic drugs, in particular for the nose-to-brain pathway. Simvastatin was selected as an ideal model drug as it has a sound rationale for its use in neurodegenerative diseases and could benefit from an alternative administration route, providing a direct access to the $\mathrm{CNS}^{63}$

In order to efficiently load simvastatin into LCNs, it was necessary to add into the formulation an oily component. Various pharmaceutical lipophilic solvents were selected based on simvastatin solubility data reported in the literature. In a systematic study of simvastatin solubility in oils and surfactants, Capryol PGMC and Capryol 90 were found to be the best solvents for simvastatin $(\sim 105 \mathrm{mg} / \mathrm{mL})$, followed by other oily excipients, such as Maisine $(\sim 60 \mathrm{mg} / \mathrm{mL})$ and Labrafac $(\sim 25 \mathrm{mg} / \mathrm{mL}) .{ }^{64}$ Therefore, combinations of these oils were used in order to optimize EE of simvastatin in LCNs. Nanoparticles with a positive surface charge and a size of around 200-300 $\mathrm{nm}$ in size were obtained, showing a good EE of simvastatin and providing a 30 -fold increase in simvastatin apparent solubility in water. Interestingly, the best formulation, containing the combination of glyceryl monolinoleate (Maisine) and medium-chain triglycerides (Labrafac), reached an apparent simvastatin solubility which is fivefold of that expected by extrapolating from the solubility of the drug in the two solvents and from their respective amounts in the formulation, showing a synergistic effect. This suggests an optimized accommodation of the simvastatin into the oily core of the particles surrounded by a lecithin/chitosan shell, as evidenced by the SAXS experiments. It could be hypothesized that Maisine, a lipid with a slightly higher melting point than the others selected (Maisine is liquid at $40^{\circ} \mathrm{C}$ ), 
can intimately mix with the molecules of Labrafac when heated, forming a semisolid oil core when cooled down at room temperature, favoring simvastatin entrapment. In fact, LCN_MaiLab showed the highest EE of formulated drug, encapsulating it almost entirely.

Concerning the stability of the preparations, it has been previously reported that surface charge is an important indicator of the stability of nanoparticles. In particular, when the zeta potential exceeds $30 \mathrm{mV}$, nanoparticles are regarded as stable, their stability increasing along with net surface charge. ${ }^{65}$ Blank nanoparticles displayed similar values of zeta potential, generally higher than their equivalent loaded nanoparticles (Table 1). The surface charge of all LCNs was highly positive, as expected due to the presence of chitosan, a positive polysaccharide covering the surface of the nanoparticles, as reported previously. The introduction of simvastatin and of some oils into LCNs resulted in a decrease in their surface charge (SVT-LCN_Cap Cap $_{\text {II }}$, SVT-LCN_MaiCap ${ }_{\mathrm{I}}$, and SVT-LCN_LabCap $)$. As the simvastatin hydroxy-acid form is capable of deprotonating to a negatively charged ion, this could lead to charge neutralization and loading close to the external shell.

On the contrary, the addition of the oil combination Maisine plus Labrafac into LCNs (SVT-LCN_MaiLab) did not result in an important reduction of the positive surface charge, in line with the already suggested efficient trapping of simvastatin in a solid lipid core. A similar behavior was also observed for SVT-LCN_Mai ${ }_{2}$, supporting the effectiveness of this oil in embedding simvastatin inside the core, thus conferring great stability to the nanoparticles system.

This core-shell organization evidenced from the nanoparticle structure investigation by SAXS is maintained upon interaction with mucus. The interaction of the superficial shell with the mucus is in agreement with a number of previous studies that indicate mucoadhesion as an important feature of nasal preparations. In particular, this may provide a much higher residence time in the nasal cavity, pivotal to allow an enhancement of drug availability. ${ }^{66}$

For the in vitro release studies, a simulated nasal electrolyte solution containing BSA was preferred to surfactant solutions ${ }^{48}$ or mixtures with organic solvents miscible with water $^{67}$, as a physiological dissolution medium. Generally, drug inclusion in nanoparticles is a strategy to prolong the release. In this study, on the contrary, after a similar initial "burst" release, a faster release rate was evidenced for nanoparticles in comparison to a simple simvastatin suspension, likely resulting from the high surface area of nanoparticles and the efficient dispersion of the drug in the nanoparticle core.
This result is even more relevant as usually, apparent drug release in dialysis methods is hindered by the dialysis membrane itself, which poses an additional barrier to the diffusion of the drug to the receiver compartment. ${ }^{68}$

As already pointed out, the major drawback affecting the nasal administration is the removal of the formulation through the mucociliary clearance, a mechanism that can reduce the bioavailability of poorly water-soluble drugs. ${ }^{52}$ Hence, fast absorption is required in order to achieve the needed therapeutic concentrations. The role of chitosan in promoting mucoadhesion and penetration is well known. Several studies suggest that chitosan nanoparticles are transported by transmucosal route with increased uptake when compared to other nanoparticles as a result of the chitosan mucoadhesivity. ${ }^{69,70}$ However, it is doubtful that slow-release nanoparticles would provide a significant improvement of intranasal drug delivery. ${ }^{71}$ For this reason, the present system, which is both mucoadhesive and fast releasing as compared to a typical formulation for nasal administration of drug suspensions, could overcome limitation on delivery due to clearance. Moreover, previous studies have shown that LCNs are highly susceptible to degradation by GI enzymes, thus promoting drug release and drug permeation through intestinal epithelium via an enhanced paracellular transport. ${ }^{31}$ This degradation is also likely to occur on the nasal mucosal surface. Degradation has been evidenced in the presence of mucus and lysozyme, where a breakdown of the original particle structure occurs over time (Figure 5). In fact, nasal secretions are rich in antibacterial peptides and proteins that are part of the innate immune defenses of the body. ${ }^{44}$ Among these proteins, one of the most abundant is lysozyme, an enzyme able to degrade proteoglycans of bacterial cell wall, but also able to degrade chitosan. ${ }^{72}$ The degradation of the nanoparticles by endogenous enzymatic process constitutes a new approach for nasal delivery and could represent an interesting Trojan-horse strategy for improving the nasal bioavailability of statins and other poorly soluble drugs.

Interestingly, despite reports of concerns in the literature regarding the biocompatibility of positively charged chitosan nanoparticles, the cytotoxicity studies carried out evidenced no apparent toxicity of the proposed drug nanocarriers. This is of course an important result, as safety is a prerequisite to the use of the formulation. In fact, the nasal epithelial layer represents one of the first body's defense lines, and materials harming the mucosal barrier constitute a potential health risk. ${ }^{73}$

The cytotoxicity evidenced by simvastatin-loaded nanoparticles is actually related to the statin itself. In fact, several 
recent studies have demonstrated the anticancer activity of statins in various cancer cells. ${ }^{10,74,75}$ Since RPMI 2650 cells are derived from an epidermoid carcinoma of the nasal septum, the cytotoxic activity detected is to be attributed to the drug released by nanoparticles or by the direct uptake of nanoparticles by the cells. As nanoparticles degradation and/or drug release is time dependent, SVT-LCN_MaiLab showed lower $\mathrm{IC}_{50}$ values than simvastatin.

In vivo preliminary studies were carried out to assess the potential of the formulation for the proposed nose-to-brain delivery of statins. Gamma scintigraphy was selected as a powerful and rapid method to evaluate the biodistribution of the formulation after nasal administration in rodents. ${ }^{76} \mathrm{~A}$ suspension of simvastatin was used as a control. It is worth pointing out that the radioactivity that is detected cannot be considered permanently bound to the particles or to the drug molecule. However, it appears clear that after the nasal instillation of the nanoparticle formulation, the radioactivity was localized mainly in the kidneys and in the brain. This biodistribution implies a significant absorption of the radiolabel through the nasal mucosa, which accounts for the localization in the kidney where the radioisotope will be eliminated by filtration and an efficient transfer via the noseto-brain pathway of around $20 \%$ of the administered radioactivity dose. This was not observed for the controls, that is, simvastatin suspension and the solution of the radioisotope itself, where the radioactivity mainly localized in the lungs and GI tract of the animals, as often happens for rodents that are obligate nose breather. It can be hypothesized that the nanoparticle formulation is more effective through both chitosan-mediated mucoadhesion ${ }^{77}$ and penetration enhancement via tight-junction opening, ${ }^{78}$ but also most likely as a consequence of mucosal biodegradation of the nanoparticle structure. Overall, these processes are able to favor the transmucosal absorption of the radioactivity, independently from the form it may assume: isotopes linked to entire nanoparticles, particle fragments, polysaccharide chains or free from linkage to any of nanoparticle structures.

\section{Conclusion}

Although some brain-targeted nanoparticles loaded with statins have been proposed by some other authors, this is to our knowledge the first research proposing nanoparticles to be administered nasally to deliver statins to the brain. The particles were designed to optimize the loading of a lipophilic drug such as simvastatin and to provide multiple features helpful for nasal delivery, such as physical and chemical stability, biocompatibility, mucoadhesion, and a rapid drug release. Furthermore, the nanoparticles appear to be prone to a mucus-specific biodegradation process that represents an innovative Trojan-horse strategy able to boost the permeation of the encapsulated drug. Preliminary in vivo gamma scintigraphy studies showed an enhanced nose-tobrain transport of the radioactivity administered nasally for the SVT-LCNs but not for a more traditional formulation such as a suspension. Although further studies are necessary to elucidate if the nanoparticles are taken up by the nasal epithelium or simply favor the drug absorption without crossing the mucosa and to investigate the pharmacokinetics and efficacy of the nanoformulated statin after administration via the nasal route, the proposed nanoformulation appears to be an optimal drug delivery platform for poorly soluble drugs that need to get administered to the nasal mucosa for systemic or CNS delivery.

\section{Acknowledgments}

Adryana Clementino and Gabriela Garrastazu would like to acknowledge the Brazilian government as recipients of CNPq grants in the program "Ciências sem Fronteiras". Fabio Sonvico would like to thank the University of Technology Sydney for the support received for his research through Early Career Researcher Grant program.

\section{Disclosure}

The authors report no conflicts of interest in this work.

\section{References}

1. Bonetti PO, Lerman LO, Napoli C, Lerman A. Statin effects beyond lipid lowering - are they clinically relevant? Eur Heart J. 2003;24(3): 225-248.

2. Heart Protection Study Collaborative Group. MRC/BHF Heart Protection Study of cholesterol lowering with simvastatin in 20,536 high-risk individuals: a randomised placebo-controlled trial. Lancet. 2002; 360(9326):7-22.

3. Romana B, Batger M, Prestidge CA, Colombo G, Sonvico F. Expanding the therapeutic potential of statins by means of nanotechnology enabled drug delivery systems. Curr Top Med Chem. 2014;14(9):1182-1193.

4. Liao JK, Laufs U. Pleiotropic effects of statins. Annu Rev Pharmacol Toxicol. 2005;45:89-118.

5. Palinski W, Napoli C. Unraveling pleiotropic effects of statins on plaque rupture. Arterioscler Thromb Vasc Biol. 2002;22(11):1745-1750.

6. Blanco-Colio LM, Tuñón J, Martín-Ventura JL, Egido J. Antiinflammatory and immunomodulatory effects of statins. Kidney Int. 2003;63(1):12-23.

7. Jain MK, Ridker PM. Anti-inflammatory effects of statins: clinical evidence and basic mechanisms. Nat Rev Drug Discov. 2005;4(12):977-987.

8. McCarey DW, McInnes IB, Madhok R, et al. Trial of Atorvastatin in Rheumatoid Arthritis (TARA): double-blind, randomised placebocontrolled trial. Lancet. 2004;363(9426):2015-2021.

9. Marin L, Colombo P, Bebawy M, Young PM, Traini D. Chronic obstructive pulmonary disease: patho-physiology, current methods of treatment and the potential for simvastatin in disease management. Expert Opin Drug Deliv. 2011;8(9):1205-1220. 
10. Gopalan A, Yu W, Sanders BG, Kline K. Simvastatin inhibition of mevalonate pathway induces apoptosis in human breast cancer cells via activation of JNK/CHOP/DR5 signaling pathway. Cancer Lett. 2013; 329(1):9-16

11. Wang Q, Yan J, Chen X, et al. Statins: multiple neuroprotective mechanisms in neurodegenerative diseases. Exp Neurol. 2011;230(1): 27-34.

12. Ghribi O, Larsen B, Schrag M, Herman MM. High cholesterol content in neurons increases BACE, $\beta$-amyloid, and phosphorylated tau levels in rabbit hippocampus. Exp Neurol. 2006;200(2):460-467.

13. Puglielli L, Tanzi RE, Kovacs DM. Alzheimer's disease: the cholesterol connection. Nat Neurosci. 2003;6(4):345-351.

14. $\mathrm{Bu} \mathrm{G}$. Apolipoprotein $\mathrm{E}$ and its receptors in Alzheimer's disease: pathways, pathogenesis and therapy. Nat Rev Neurosci. 2009;10(5): 333-344.

15. Kivipelto M, Helkala EL, Laakso MP, et al. Apolipoprotein E epsilon4 allele, elevated midlife total cholesterol level, and high midlife systolic blood pressure are independent risk factors for late-life Alzheimer disease. Ann Intern Med. 2002;137(3):149-155

16. Fassbender K, Simons M, Bergmann C, et al. Simvastatin strongly reduces levels of Alzheimer's disease $\beta$-amyloid peptides $A \beta 42$ and $A \beta 40$ in vitro and in vivo. Proc Natl Acad Sci U S A. 2001;98(10):5856-5861.

17. Ehehalt R, Keller P, Haass C, Thiele C, Simons K. Amyloidogenic processing of the Alzheimer $\beta$-amyloid precursor protein depends on lipid rafts. J Cell Biol. 2003;160(1):113-123.

18. Schachter M. Chemical, pharmacokinetic and pharmacodynamic properties of statins: an update. Fundam Clin Pharmacol. 2005;19(1): $117-125$.

19. Pires A, Fortuna A, Alves G, Falcão A. Intranasal drug delivery: how, why and what for? J Pharm Pharm Sci. 2009;12(3):288-311.

20. Suman JD. Current understanding of nasal morphology and physiology as a drug delivery target. Drug Deliv Transl Res. 2013;3(1):4-15.

21. Mistry A, Glud SZ, Kjems J, et al. Effect of physicochemical properties on intranasal nanoparticle transit into murine olfactory epithelium. J Drug Target. 2009;17(7):543-552.

22. Kozlovskaya L, Abou-Kaoud M, Stepensky D. Quantitative analysis of drug delivery to the brain via nasal route. J Control Release. 2014; 189:133-140.

23. Tayebati SK, Nwankwo IE, Amenta F. Intranasal drug delivery to the central nervous system: present status and future outlook. Curr Pharm Des. 2013;19(3):510-526.

24. Illum L. Transport of drugs from the nasal cavity to the central nervous system. Eur J Pharm Sci. 2000;11(1):1-18.

25. Illum L. Is nose-to-brain transport of drugs in man a reality? J Pharm Pharmacol. 2004;56(1):3-17.

26. Horvát S, Fehér A, Wolburg H, et al. Sodium hyaluronate as a mucoadhesive component in nasal formulation enhances delivery of molecules to brain tissue. Eur J Pharm Biopharm. 2009;72(1):252-259.

27. $\mathrm{Wu} \mathrm{H}, \mathrm{Hu} \mathrm{K}$, Jiang $\mathrm{X}$. From nose to brain: understanding transport capacity and transport rate of drugs. Expert Opin Drug Deliv. 2008;5(10):1159-1168.

28. Zhang QZ, Zha LS, Zhang Y, et al. The brain targeting efficiency following nasally applied MPEG-PLA nanoparticles in rats. J Drug Target. 2008;14(5):281-290.

29. Betbeder D, Spérandio S, Latapie JP, et al. Biovector nanoparticles improve antinociceptive efficacy of nasal morphine. Pharm Res. 2000; 17(6):743-748.

30. Gerelli Y, Di Bari MT, Deriu A, et al. Structure and organization of phospholipid/polysaccharide nanoparticles. JPhys Condens Matter. 2008; 20(10):104211.

31. Barbieri S, Buttini F, Rossi A, et al. Ex vivo permeation of tamoxifen and its $4-\mathrm{OH}$ metabolite through rat intestine from lecithin/chitosan nanoparticles. Int J Pharm. 2015;491(1-2):99-104.

32. Takeuchi H, Matsui Y, Yamamoto H, Kawashima Y. Mucoadhesive properties of carbopol or chitosan-coated liposomes and their effectiveness in the oral administration of calcitonin to rats. J Control Release. 2003;86(2-3):235-242.
33. Smith JM, Dornish M, Wood EJ. Involvement of protein kinase C in chitosan glutamate-mediated tight junction disruption. Biomaterials. 2005;26(16):3269-3276.

34. Hole P, Sillence K, Hannell C, et al. Interlaboratory comparison of size measurements on nanoparticles using nanoparticle tracking analysis (NTA). J Nanopart Res. 2013;15(12):2101.

35. Filipe V, Hawe A, Jiskoot W. Critical evaluation of Nanoparticle Tracking Analysis (NTA) by NanoSight for the measurement of nanoparticles and protein aggregates. Pharm Res. 2010;27(5):796-810.

36. Yang DJ, Hwang LS. Study on the conversion of three natural statins from lactone forms to their corresponding hydroxy acid forms and their determination in Pu-Erh tea. J Chromatogr A. 2006;1119(1-2):277-284.

37. Castile J, Cheng YH, Simmons B, Perelman M, Smith A, Watts P. Development of in vitro models to demonstrate the ability of PecSys ${ }^{\circledR}$, an in situ nasal gelling technology, to reduce nasal run-off and drip. Drug Dev Ind Pharm. 2013;39(5):816-824.

38. Martins FP, Gutfilen B, de Souza SA, et al. Monitoring rheumatoid arthritis synovitis with 99mTc-anti-CD3. Br J Radiol. 2008; 81(961):25-29.

39. Martins FP, Souza SA, Gonçalves RT, Fonseca LM, Gutfilen B. Preliminary results of [99mTc]OKT3 scintigraphy to evaluate acute rejection in renal transplants. Transplant Proc. 2004;36(9):2664-2667.

40. Lopes FP, de Azevedo MN, Marchiori E, da Fonseca LM, de Souza SA, Gutfilen B. Use of $99 \mathrm{mTc}$-anti-CD3 scintigraphy in the differential diagnosis of rheumatic diseases. Rheumatology (Oxford). 2010;49(5): 933-939.

41. Gerelli Y, Di Bari MT, Barbieri S, et al. Flexibility and drug release features of lipid/saccharide nanoparticles. Soft Matter. 2010;6(3): 685-691.

42. Wright M. Nanoparticle tracking analysis for the multiparameter characterization and counting of nanoparticle suspensions. Methods Mol Biol. 2012;906:511-524.

43. Sokolova V, Ludwig AK, Hornung S, et al. Characterisation of exosomes derived from human cells by nanoparticle tracking analysis and scanning electron microscopy. Colloids Surf B Biointerfaces. 2011;87(1):146-150.

44. Travis SM, Singh PK, Welsh MJ. Antimicrobial peptides and proteins in the innate defense of the airway surface. Curr Opin Immunol. 2001; 13(1):89-95.

45. Cole AM, Dewan P, Ganz T. Innate antimicrobial activity of nasal secretions. Infect Immun. 1999;67(7):3267-3275.

46. D'Souza SS, DeLuca PP. Methods to assess in vitro drug release from injectable polymeric particulate systems. Pharm Res. 2006; 23(3):460-474.

47. Chen KH, Di Sabatino M, Albertini B, Passerini N, Kett VL. The effect of polymer coatings on physicochemical properties of spray-dried liposomes for nasal delivery of BSA. Eur J Pharm Sci. 2013;50(3-4):312-322.

48. Sood S, Jain K, Gowthamarajan K. Optimization of curcumin nanoemulsion for intranasal delivery using design of experiment and its toxicity assessment. Colloids Surf B Biointerfaces. 2014;113:330-337.

49. Seju U, Kumar A, Sawant KK. Development and evaluation of olanzapine-loaded PLGA nanoparticles for nose-to-brain delivery: in vitro and in vivo studies. Acta Biomater. 2011;7(12):4169-4176.

50. Bai S, Yang T, Abbruscato TJ, Ahsan F. Evaluation of human nasal RPMI 2650 cells grown at an air-liquid interface as a model for nasal drug transport studies. J Pharm Sci. 2008;97(3):1165-1178.

51. Lochhead JJ, Thorne RG. Intranasal delivery of biologics to the central nervous system. Adv Drug Deliv Rev. 2012;64(7):614-628.

52. Sigurdsson HH, Kirch J, Lehr CM. Mucus as a barrier to lipophilic drugs. Int J Pharm. 2013;453(1):56-64

53. Mistry A, Stolnik S, Illum L. Nanoparticles for direct nose-to-brain delivery of drugs. Int J Pharm. 2009;379(1):146-157.

54. Kumar A, Pandey AN, Jain SK. Nasal-nanotechnology: revolution for efficient therapeutics delivery. Drug Deliv. 2016;23(3):681-693.

55. Bernkop-Schnürch A, Dünnhaupt S. Chitosan-based drug delivery systems. Eur J Pharm Biopharm. 2012;81(3):463-469. 
56. Akbarzadeh A, Rezaei-Sadabady R, Davaran S, et al. Liposome: classification, preparation, and applications. Nanoscale Res Lett. 2013;8(1):102.

57. Gabizon AA, Shmeeda H, Zalipsky S. Pros and cons of the liposome platform in cancer drug targeting. J Liposome Res. 2006;16(3):175-183.

58. Barenholz Y. Doxil ${ }^{\circledR}$ - the first FDA-approved nano-drug: lessons learned. J Control Release. 2012;160(2):117-134.

59. Di Bari MT, Gerelli Y, Sonvico F, et al. Dynamics of lipid-saccharide nanoparticles by quasielastic neutron scattering. Chem Phys. 2008; 345(2-3):239-244.

60. Sonvico F, Di Bari MT, Bove L, Deriu A, Cavatorta F, Albanese G. Mean square hydrogen fluctuations in chitosan/lecithin nanoparticles from elastic neutron scattering experiments. Physica B. 2006; 385-386:725-727.

61. Senyiğit T, Sonvico F, Barbieri S, Ozer O, Santi P, Colombo P. Lecithin/ chitosan nanoparticles of clobetasol-17-propionate capable of accumulation in pig skin. J Control Release. 2010;142(3):368-373.

62. Barbieri S, Sonvico F, Como C, et al. Lecithin/chitosan controlled release nanopreparations of tamoxifen citrate: loading, enzyme-trigger release and cell uptake. J Control Release. 2013;167(3):276-283.

63. Jiang Y, Li Y, Liu X. Intranasal delivery: circumventing the iron curtain to treat neurological disorders. Expert Opin Drug Deliv. 2015; 12(11):1717-1725.

64. Karim FT, Kalam A, Anwar R, Miah MM, Rahman MS, Islam SM. Preparation and evaluation of SEDDS of simvastatin by in vivo, in vitro and ex vivo technique. Drug Dev Ind Pharm. 2014;41(8):1338-1342.

65. Müller RH, Jacobs C, Kayser O. Nanosuspensions as particulate drug formulations in therapy. Rationale for development and what we can expect for the future. Adv Drug Deliv Rev. 2001;47(1):3-19.

66. Casettari L, Illum L. Chitosan in nasal delivery systems for therapeutic drugs. J Control Release. 2014;190:189-200.

67. Dalpiaz A, Ferraro L, Perrone D, et al. Brain uptake of a Zidovudine prodrug after nasal administration of solid lipid microparticles. Mol Pharm. 2014;11(5):1550-1561.
68. Modi S, Anderson BD. Determination of drug release kinetics from nanoparticles: overcoming pitfalls of the dynamic dialysis method. Mol Pharm. 2013;10(8):3076-3089.

69. Artursson P, Lindmark T, Davis SS, Illum L. Effect of chitosan on the permeability of monolayers of intestinal epithelial cells (Caco-2). Pharm Res. 1994;11(9):1358-1361.

70. Prego C, García M, Torres D, Alonso MJ. Transmucosal macromolecular drug delivery. J Control Release. 2005;101(1-3):151-162.

71. Illum L. Nanoparticulate systems for nasal delivery of drugs: a real improvement over simple systems? J Pharm Sci. 2007;96(3): 473-483.

72. Nordtveit RJ, Vårum KM, Smidsrød O. Degradation of partially $\mathrm{N}$-acetylated chitosans with hen egg white and human lysozyme. Carbohydr Polym. 1996;29(2):163-167.

73. Ekelund K, Östh K, Påhlstorp C, Björk E, Ulvenlund S, Johansson F. Correlation between epithelial toxicity and surfactant structure as derived from the effects of polyethyleneoxide surfactants on caco- 2 cell monolayers and pig nasal mucosa. J Pharm Sci. 2005;94(4):730-744.

74. Wu H, Jiang H, Lu D, et al. Effect of simvastatin on glioma cell proliferation, migration, and apoptosis. Neurosurgery. 2009;65(6):1087-1096; discussion 1096-1097.

75. Ali H, Shirode AB, Sylvester PW, Nazzal S. Preparation, characterization, and anticancer effects of simvastatin-tocotrienol lipid nanoparticles. Int J Pharm. 2010;389(1-2):223-231.

76. Alam S, Khan ZI, Mustafa G, et al. Development and evaluation of thymoquinone-encapsulated chitosan nanoparticles for nose-tobrain targeting: a pharmacoscintigraphic study. Int J Nanomedicine. 2012;7:5705-5718.

77. Jogani VV, Shah PJ, Mishra P, Mishra AK, Misra AR. Intranasal mucoadhesive microemulsion of tacrine to improve brain targeting. Alzheimer Dis Assoc Disord. 2008;22(2):116-124.

78. Vllasaliu D, Exposito-Harris R, Heras A, et al. Tight junction modulation by chitosan nanoparticles: comparison with chitosan solution. Int J Pharm. 2010;400(1-2):183-193.
International Journal of Nanomedicine

\section{Publish your work in this journal}

The International Journal of Nanomedicine is an international, peerreviewed journal focusing on the application of nanotechnology in diagnostics, therapeutics, and drug delivery systems throughout the biomedical field. This journal is indexed on PubMed Central, MedLine, CAS, SciSearch ${ }^{\circledR}$, Current Contents ${ }^{\circledR} /$ Clinical Medicine,

\section{Dovepress}

Journal Citation Reports/Science Edition, EMBase, Scopus and the Elsevier Bibliographic databases. The manuscript management system is completely online and includes a very quick and fair peer-review system, which is all easy to use. Visit http://www.dovepress.com/ testimonials.php to read real quotes from published authors. 